\title{
Implementing the World Health Organization Global Strategy on Diet, Physical Activity and Health
}

\section{Colin Tukuitonga and Ingrid Keller}

Department of Chronic Diseases and Health Promotion, World Health Organization, Geneva, Switzerland

\begin{abstract}
The World Health Organization (WHO) Global Strategy on Diet, Physical Activity and Health (DPAS) was adopted by the World Health Assembly in May 2004 (Resolution 57.17). Implementing DPAS is challenging because current investment in chronic disease prevention and control at national and international levels seriously lags behind what is required, and variable levels of political commitment lead to a limited capacity to respond effectively. Nevertheless, WHO Resolutions are valuable advocacy tools and, thus, DPAS presents a golden opportunity to develop national chronic disease prevention and control policies, plans and programmes where none exists, or to strengthen existing national responses where these are already in existence. WHO is also using the opportunity to engage all stakeholders in aligning their plans and activities with the objectives of DPAS. Non-governmental organizations, the Codex Alimentarius Commission, relevant WHO Collaborating Centres and the private sector (especially the food and non-alcoholic beverage drinks manufacturers and advertisers) are particularly important. Some activities are undertaken at the global level, e.g. interactions with Codex, and others are undertaken at the regional office level, e.g. providing technical support to countries. A critical aspect of the implementation process involves the production of suitable tools - guidelines, frameworks, protocols and good practice models - in a range of areas, to assist member states with the implementation of DPAS.
\end{abstract}

Received: 24 Jul. 2005; Accepted: 26 Jul. 2005

\section{Introduction}

The World Health Organization (WHO) Global Strategy on Diet, Physical Activity and Health (DPAS) was adopted by the World Health Assembly (WHA) in May 2004. This paper presents an overview of the implementation of the Strategy, key challenges and measures that are needed to ensure that the Strategy is used to strengthen and advance chronic disease prevention and control.

\section{Background}

In response to the global epidemic of chronic, noncommunicable diseases (NCDs), the WHA in 2002 requested the WHO to develop the Global Strategy on Diet, Physical Activity and Health to strengthen existing national, regional and international efforts to prevent and control chronic diseases and their common risk factors. DPAS was formally adopted at the WHA in May 2004 (Resolution 57.17) (1)

Despite active opposition by the sugar industry and some countries, DPAS was universally endorsed by the WHO member states $(2,3)$. The adoption of DPAS (and the landmark Framework Convention on Tobacco Control adopted by the WHA in 2003), represents a significant development in the global effort to prevent and control chronic diseases and their common risk factors. Experiences from a number of developed countries show that if the key objectives of these policy instruments are implemented, a significant proportion of the leading chronic diseases and their common risk factors will be prevented. In addition, provision of low-cost evidence-based therapeutic interventions for people with established chronic diseases will result in significant social and economic benefits to individuals and societies.

DPAS is a comprehensive set of policy options that countries can adopt and implement according to their national circumstances. It recognizes that only a long-term, multistakeholder and society-wide approach can counteract this worldwide public health problem, and that actions are needed at all 
levels: global, regional, national, community and individual.

Policy options are directed at WHO, member states, international partners, civil society and nongovernmental organizations and the private sector. Key DPAS principles are to tackle the rapid worldwide increase in chronic diseases through primary prevention, targeting their common risk factors (unhealthy diets and physical inactivity), working through a multisectoral approach to prevention by involving, under the leadership of the health sector, other sectors such as agriculture, urban planning and transport, sports, education, trade and economic development.

\section{The challenges of implementation}

The major challenges to the implementation of DPAS are due mainly to political and financial constraints, because enough is known about what works and suitable tools are available for the prevention and control of chronic diseases. The level of political commitment is highly variable, and thus there is inadequate investment in chronic disease prevention and control and the capacity to respond effectively is limited at international and national levels.

Nevertheless, WHO Resolutions are valuable advocacy tools in highlighting global public health problems and enabling member states to strengthen public health interventions in relevant areas. Despite the rapid escalation in obesity, chronic diseases and their common risk factors in all parts of the world, global investment in chronic disease prevention and control has lagged behind in most countries. Resolution 57.17 provides a unique opportunity to advance the global mission to prevent and control chronic diseases.

Common misconceptions about chronic diseases mean that individuals responsible for resource allocation decisions are not adequately informed of the economic and social consequences of these diseases. Not only do chronic diseases present barriers to national development activities, but these conditions are both causes and effects of poverty and poor health at the personal level. Unlike many acute infectious conditions where outcomes are usually known in a short period, people affected by chronic diseases often have prolonged poor quality of life, living with one or more chronic diseases and their complications.
Within WHO, the organization currently spends less than $3 \%$ of its budget on chronic diseases, despite the fact that these conditions cause $60 \%$ of the global mortality annually. Consequently, the current chronic disease prevention and control capacity within the organization is seriously limited, especially at the regional offices, where the human resource capacity is committed to many existing demands. This is a serious challenge because most of the support needed for implementation of DPAS at the national level will rely on actions taken at the regional office level. Resource constraints at all levels compromise the ability of WHO to provide the technical support needed by member states and the ability to advance the normative work needed to support implementation.

Therefore, a key part of the implementation of DPAS is mobilizing resources at all levels. Resource mobilization includes securing financial as well as human resources, through recruitment or secondment of suitable experts to areas of need. Assistance could also be directed at the development of suitable tools and completion of other normative functions such as guideline development and providing the evidence base for actions. An essential element in expanding the WHO resource base is the development of appropriate interactions with suitable stakeholders in areas of mutual benefit.

\section{WHO secretariat's approach to the implementation of DPAS}

The WHO's approach to the implementation of DPAS involves two main streams of work: (i) activities at the WHO regional offices to support implementation at country level; and (ii) activities at the global level to address issues best managed at the international level, e.g. interactions with the global private sector, United Nations (UN) agencies, work with the Codex Alimentarius Commission (CAC) and normative functions.

In promoting the implementation of DPAS, every effort is made to use existing WHO activities by relevant units and programmes for improved synergy. Several WHO units are involved, but the Department of Nutrition for Health Development plays a central role in the implementation of DPAS. Similarly, the Family and Child Health and the Sustainable Development and Environments Clusters make valuable contributions. The WHO Country Cooperation Strategy is an important 
mechanism for incorporating DPAS objectives into national plans.

\section{Activities at the regional level}

Some member states have existing plans, policies and programmes for the prevention and control of chronic diseases and their common risk factors. These include NCD or disease-specific policies and plans, management guidelines, food-based dietary guidelines and tobacco control activities.

The impact of many of these initiatives has, so far, been limited, and therefore DPAS presents a unique opportunity to strengthen chronic disease prevention and control plans and programmes where these already exist. Where no such policies, plans or programmes exist, WHO expects that member states will seriously consider using the adoption of Resolution 57.17 to develop national responses appropriate to local circumstances using DPAS as the framework for action.

The WHO secretariat will continue to encourage and support all countries to consider implementing all or part of DPAS, and especially countries that already experience a high burden of mortality and morbidity from these diseases. In some areas, regional and subregional approaches can provide additional cross-border measures to help to strengthen national plans and programmes. Regional or subregional interventions in advocacy, trade, training, research and interactions with the private sector may be more efficient and effective than every single nation attempting to do so at the national level.

The WHO regional offices will adopt approaches as appropriate for their regions member states, e.g. in the Western Pacific a subregional plan has been developed for the Pacific Islands to support national activities in those countries.

An innovative development at the regional level is the initiative taken by the European Union (EU) on interactions with the private sector (4). The EU Platform on Diet, Physical Activity and Health is an important development whereby the EU is facilitating the contributions from various interested stakeholders towards the achievement of the objectives of DPAS. The aim is to facilitate voluntary but measurable actions by stakeholders to improve diet and physical activity levels in populations of the European region. This initiative supports similar activities at the national level and complements global activities in this area.
While the WHO secretariat will continue to encourage and support as many member states as possible to implement the objectives of DPAS, the responsibility for action rests primarily with member states. Although DPAS policies are directed at all levels and audiences, governments have a central stewardship and leadership role, and ministries of health need to facilitate and co-ordinate the contribution from all stakeholders towards the prevention and control of chronic diseases and their common risk factors.

Good examples of national developments have emerged. In response to the rising obesity epidemic in Spain and the adoption of the DPAS, the Agencia Española de Seguridad Alimentaria (AESA; Spanish Food Safety Agency) has developed the NAOS Strategy for the prevention of obesity in that country (5). It was developed through a multisectoral process involving all stakeholders, with strong representation from the private sector. Similar developments are taking place elsewhere.

\section{Activities at the global level}

Global activities are directed mainly at issues most efficiently addressed at the international level. These include interactions with global food and soft drink manufacturers, sporting goods and physical activity entities, advertisers, the CAC and UN agencies, and normative functions such as the development of suitable tools for member states.

DPAS recognizes the need for all stakeholders to contribute to the creation of supportive environments for individuals. It advocates effective interactions with the private sector, especially the food and soft drink industries, sporting goods and related physical activity organizations, marketing and advertising groups. Considerable progress has been made by many global food and soft drink companies in product formulation and reformulation, improving consumer choices and information, but an industry-wide response is still needed. Furthermore, the retail and catering sectors and restaurants need to do more to improve their products and information for consumers. Similarly, marketing and advertising practices are improving, but most existing codes of practice are industry owned and governed. Many member states have requested assistance from WHO to develop more objective and independent marketing codes of practice. Similarly, countries need guidance on interacting 
appropriately with the private sector to protect and promote public health, and reduce the risks of chronic diseases.

DPAS also calls for co-ordinated and collaborative work with international organizations, and has requested investigation of the use of international standards to support the objectives of DPAS. In the food area, the work of the CAC is relevant. The WHA Resolution endorsing DPAS specifically requested the "Codex Alimentarius Commission to continue to give full consideration, within the framework of its operational mandate, to evidencebased action it might take to improve the health standards of foods consistent with the aims and objectives of the strategy". The aim is to explore how the Codex Alimentarius, as the system setting food standards, guidelines and related texts to protect the health of consumers and ensure fair trade practices, can support and contribute to the implementation of DPAS.

The work of the CAC on nutrition labelling, nutrition and health claims provides important information about the nutrient content of foods and the role of diet in the reduction of diseases. DPAS specifically refers to the Codex Guidelines on Nutrition Labelling, specifying that "consumers require accurate, standardized and comprehensible information on the content of food items in order to make healthy choices", and suggesting that governments may require information to be provided on key nutritional aspects. With respect to health claims, DPAS requests that these messages "must not mislead the public about nutritional benefits or risks".

DPAS also recommends that agricultural policies at international and national levels should be supportive of public health objectives. The Food and Agriculture Organization (FAO) plays a critical role in this respect through its their advice to member states. Although this opportunity to influence agricultural policies remains largely unexplored, WHO and FAO in association with the WHO Kobe Centre for Health Development jointly developed the FAO/WHO Framework to promote increased production and consumption of fruit and vegetables globally (6). The health benefits of fruit and vegetable consumption are well established, but intake remains well below recommended levels. This framework provides the basis for national activities in this area.
Member states also require assistance with guidelines for interactions with the private sector, in view of the need to adopt inclusive multisectoral approaches to chronic disease prevention and control. WHO is developing suitable guidance to enable member states to engage effectively with these stakeholders, while protecting the integrity and regulatory independence of national governments.

\section{Normative functions}

WHO is the specialized agency of the UN system responsible for developing global public health policies and providing technical support for member states. One of its key functions is the development of standards, guidelines, protocols and recommendations to assist member states in the development of national policies, standards and guidelines.

WHO, in association with FAO and other UN agencies, has produced a comprehensive set of guidelines for improving population nutrition standards and food security, but very few countries have developed evidence-based guidelines for physical activity to support the development of national programmes in this area. Furthermore, levels and patterns of physical activity in developing countries differ markedly from those in developed countries, although it is widely known that overall physical activity levels have declined in all areas of the world. In response, WHO will develop and disseminate suitable guidelines for physical activity in population groups, including those for children and young people as a contribution to the prevention of childhood obesity.

Other priority areas of normative work at the global level include the development of policy frameworks and guidelines for preventing chronic diseases using the school setting, responsible marketing of food and soft drinks to children, development of planning models and frameworks for use by member states in formulating national chronic disease prevention and control policies, plans and programmes, and the development of suitable monitoring methods and indicators. WHO will also continue to promote the implementation of the WHO/FAO Framework for promoting the production and consumption of fruit and vegetables in member states. 


\section{Conclusions}

Resolution 57.17 adopting DPAS provides a renewed impetus and opportunity to strengthen international and national efforts to prevent and control chronic diseases and their common risk factors. Building on past achievements and in addition to other WHO activities in this area (such as the Global Report on Chronic Diseases, due in October 2005), there is promise and potential for significant gains to be made in halting and reversing the global epidemic.

While there are significant political and financial constraints to the implementation of DPAS, developments to date suggest that good progress has been made and further improvements will be made in combating this global public health problem. Political decisions will be made at the national level, but the international community can do more to strengthen the institutional capacity of WHO to respond to member states.

WHO will continue to develop and disseminate suitable tools and evidence-based guidelines in various areas to support member states on request. Member states are encouraged to use the opportunity created by DPAS to make progress and strengthen their national capacities for action to prevent and control chronic diseases and their common risk factors.

\section{References}

1. World Health Organization. Global Strategy on Diet, Physical Activity and Health (Resolution 57.17) [online]. Geneva: WHO; 2004. Available from: www.who.int/ dietphysicalactivity

2. Norum KR. World Health Organization's Global Strategy on Diet, Physical Activity and Health: the process behind the scenes. Scand J Nutr 2005; 49: $83-8$.

3. Asp N-G. Confrontation or collaboration? Scand J Nutr 2005; 49: 89-90.

4. European Commission. 2005 [online]. Available from: europa.eu.int/comm/health/ph_determinants/life_style/ nutrition/platform/platform_en.htm

5. Estrategia NAOS. Spanish Strategy for Nutrition, Physical Activity and Prevention of Obesity. Madrid: Agencia Española de Seguridad Alimentaria; 2005.

6. World Health Organization/Food and Agriculture Organization. Joint FAO/WHO Workshop on Fruits and Vegetables for Health, Kobe; 2004 [online report]. Available from: www.who.int/dietphysicalactivity/fruit/en

Dr Colin Tukuitonga, MPH

Primary Prevention of Chronic Diseases

Department of Chronic Diseases and Health Promotion

20 Avenue Appia

$\mathrm{CH}-12$ II Geneva

Switzerland

E-mail: tukuitongac@who.int 